\title{
Changes of hemodynamic and cerebral oxygenation after exercise in normobaric and hypobaric hypoxia: associations with acute mountain sickness
}

Tobias Kammerer ${ }^{1,25^{*}}$ D, Valentina Faihs ${ }^{1}$, Nikolai Hulde ${ }^{5}$, Andreas Bayer ${ }^{1}$, Max Hübner ${ }^{1,2}$, Florian Brettner ${ }^{1,2}$, Walter Karlen ${ }^{4}$, Julia Maria Kröpfl', Markus Rehm', Christina Spengler ${ }^{3}$ and Simon Thomas Schäfer ${ }^{1,2}$

\begin{abstract}
Objective: Normobaric $(\mathrm{NH})$ and hypobaric hypoxia $(\mathrm{HH})$ are associated with acute mountain sickness (AMS) and cognitive dysfunction. Only few variables, like heart-rate-variability, are correlated with AMS. However, prediction of AMS remains difficult. We therefore designed an expedition-study with healthy volunteers in $\mathrm{NH} / \mathrm{HH}$ to investigate additional non-invasive hemodynamic variables associated with AMS.

Methods: Eleven healthy subjects were examined in $\mathrm{NH}\left(\mathrm{FiO}_{2}\right.$ 13.1\%; equivalent of $3.883 \mathrm{~m}$ a.s.l; duration $4 \mathrm{~h}$ ) and $\mathrm{HH}$ (3.883 m a.s.l.; duration $24 \mathrm{~h}$ ) before and after an exercise of $120 \mathrm{~min}$. Changes in parameters of electrical cardiometry (cardiac index (Cl), left-ventricular ejection time (LVET), stroke volume (SV), index of contractility (ICON)), near-infrared spectroscopy (cerebral oxygenation, $\mathrm{rSCO}_{2}$ ), Lake-Louise-Score (LLS) and cognitive function tests were assessed. One-WayANOVA, Wilcoxon matched-pairs test, Spearman's-correlation-analysis and Student's t-test were performed.

Results: $\mathrm{HH}$ increased heart rate (HR), mean arterial pressure (MAP) and $\mathrm{Cl}$ and decreased LVET, SV and ICON, whereas $\mathrm{NH}$ increased HR and decreased LVET. In both NH and HH cerebral oxygenation decreased and LLS increased significantly. After $24 \mathrm{~h}$ in HH, 6 of 11 subjects (54.6\%) developed AMS. LLS remained increased until $24 \mathrm{~h}$ in $\mathrm{HH}$, whereas cognitive function remained unaltered. In HH, HR and LLS were inversely correlated $(r=-0.692 ; p<0.05)$. More importantly, the rScO2-decrease after exercise in $\mathrm{NH}$ significantly correlated with LLS after $24 \mathrm{~h}$ in $\mathrm{HH}(r=-0.971$; $p<0.01)$ and $\mathrm{rScO} 2$ correlated significantly with HR $(r=0.802 ; p<0.01), \mathrm{Cl}(r=0.682 ; p<0.05)$ and SV $(r=0.709$; $p<0.05)$ after exercise in $\mathrm{HH}$.

Conclusions: Both acute $\mathrm{NH}$ and $\mathrm{HH}$ altered hemodynamic and cerebral oxygenation and induced AMS. Subjects, who adapted their $\mathrm{Cl}$ had higher rScO2 and lower LLS. Furthermore, rScO2 after exercise under normobaric conditions was associated with AMS at high altitudes.
\end{abstract}

Keywords: Acute mountain sickness, Cerebral oxygenation, Near-infrared spectroscopy, Normobaric hypoxia, Hypobaric hypoxia, Cognitive dysfunction

\footnotetext{
*Correspondence: tobias.kammerer@med.uni-muenchen.de

'Department of Anaesthesiology, University Hospital, LMU Munich,

Marchioninistr. 15, 81377 Munich, Germany

${ }^{2}$ Walter Brendel Centre of Experimental Medicine, LMU Munich,

Marchioninistr. 15, 81377 Munich, Germany

Full list of author information is available at the end of the article
}

(c) The Author(s). Open Access This article is distributed under the terms of the Creative Commons Attribution 4.0 International License (http://creativecommons.org/licenses/by/4.0/), which permits unrestricted use, distribution, and reproduction in any medium, provided you give appropriate credit to the original author(s) and the source, provide a link to the Creative Commons license, and indicate if changes were made. The Creative Commons Public Domain Dedication waiver (http://creativecommons.org/publicdomain/zero/1.0/) applies to the data made available in this article, unless otherwise stated. 


\section{Introduction}

Acute hypoxia under both normobaric (NH) and hypobaric $(\mathrm{HH})$ conditions is associated with symptoms of acute mountain sickness (AMS) and cognitive dysfunction in humans [1-4]. The degree of hypoxemia plays a central role in the pathophysiology of AMS [5]. However, decrease of peripheral oxygen saturation (SpO2) under hypoxic conditions has previously been shown to be of poor predictive value. Therefore, most publications identified a combination of different variables to predict AMS [6, 7]. Unfortunately, some of these variables are difficult to raise under laboratory conditions or must be measured invasively. Recently, heart rate variability (HRV) was identified as a potential predictor for AMS in healthy subjects, where the underlying mechanism is unclear [8]. Predicting the likelihood to develop AMS before ascent to $\mathrm{HH}$ could be important not only for mountaineers but also for untrained individuals as improved transport technologies allow to rapidly ascending to high altitude. This exposes also persons with potentially preexisting conditions like cardiovascular disorders to an increased risk for AMS. It is therefore of particular interest to find further non-invasive variables for AMS prediction. Simultaneously, exposure to high altitudes is associated with a decrease of cerebral oxygen saturation, which is controversially discussed in terms of the incidence of cognitive dysfunction [3, 9-11].

We therefore performed a study with healthy volunteers to identify non-invasive variables under $\mathrm{NH}$ as predictors for AMS. Using electrical cardiometry, near-infrared spectroscopy, cognitive function testing and Lake-Louis-Score (LLS) we hypothesized that 1.) $\mathrm{NH}$ and $\mathrm{HH}$ would lead to similar changes of hemodynamic variables, decreases in systemic $(\mathrm{SpO} 2)$ and cerebral oxygen saturation $(\mathrm{rScO} 2)$ and that 2.) hemodynamic changes and $\mathrm{rScO}_{2}$ in $\mathrm{NH}$ would correlate with the degree of AMS in $\mathrm{HH}$.

\section{Materials and methods}

\section{Subjects and experimental protocol}

After approval by the local Ethics Committee of the University of Munich, Germany (project no. 350-16) and obtaining written informed consent, 11 healthy female $(n=5)$ and male $(n=6)$ individuals aged 36.4 $( \pm 7)$ years, with mean height of $178( \pm 6) \mathrm{cm}$ and mean body mass index of $22.7( \pm 2) \mathrm{kg} / \mathrm{m}^{2}$, were included in the study. All subjects were in good physical and mental condition, without any comorbidities or medication and were measured at different time points in normobaric normoxia, $\mathrm{NH}$ and $\mathrm{HH}$ (see Fig. 1). All individuals did not stay at a height of more than $2000 \mathrm{~m}$ a.s.l. until at least 6 weeks before the study.

The following protocol was done to evaluate effects of hypobaric hypoxia: after initial baseline measurements in Munich at $520 \mathrm{~m}$ a.s.l. (normoxia), all individuals were transferred to Zermatt, Switzerland (1608 $\mathrm{m}$ a.s.l.) by car. Next morning, ascent to Little Matterhorn at $3883 \mathrm{~m}$ a.s.l. was done by cable car (duration $45 \mathrm{~min}$ ), followed by further measurements. After this, all subjects performed

- Normobaric hypoxia

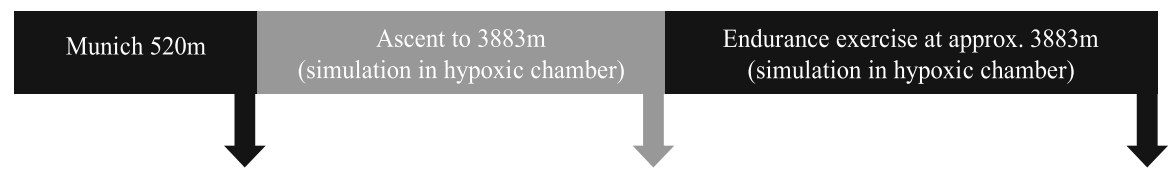

- Hypobaric hypoxia

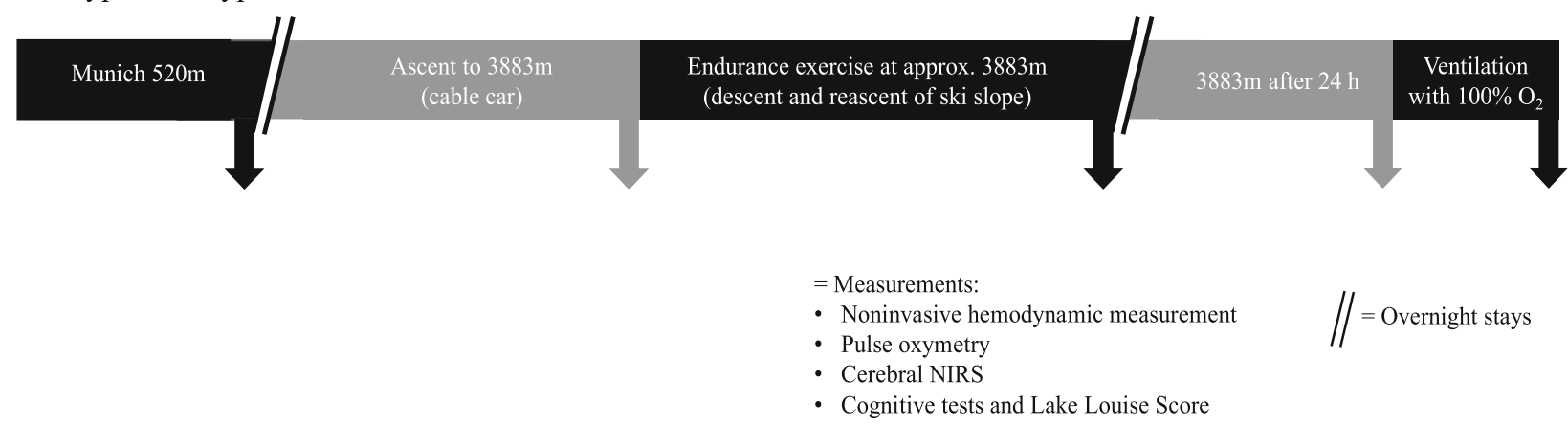

Fig. 1 Experimental protocol for ascent and exercise in normobaric (hypoxic chamber) and hypobaric (Little Matterhorn summit) hypoxia 
$120 \mathrm{~min}$ endurance exercise by descending to around $3500 \mathrm{~m}$ and reascending to $3883 \mathrm{~m}$ a.s.l.. Immediately after physical exercise, measurements were performed in an expedition tent (Keron 4 GT, Hilleberg AB, Frösön, Sweden) on the glacier. After spending one night at $3883 \mathrm{~m}$ a.s.l. in the hut, measurements were performed again $24 \mathrm{~h}$ after arrival at high altitude and repeated after breathing $100 \%$ oxygen for 5 min (Fig. 1).

Additionally, 7 of these individuals ( 3 female, 4 male; $36.3( \pm 4)$ years; $179( \pm 6) \mathrm{cm}$; BMI $\left.22.7( \pm 2) \mathrm{kg} / \mathrm{m}^{2}\right)$ were examined under normobaric conditions in a hypoxic chamber (VPSA 16; Van Amerongen CA Technology, Tiel, Netherlands) 6 weeks before $(n=4)$ and 6 weeks after $(n=3)$ high altitude exposure. Again, baseline measurements were performed in Munich at $520 \mathrm{~m}$ a.s.l. (normoxia), followed by passive ascent (duration $45 \mathrm{~min}$ ) to simulated $3883 \mathrm{~m}$ a.s.l. in the hypoxic chamber and $120 \mathrm{~min}$ of endurance exercise at simulated $3883 \mathrm{~m}$ a.s.l. including alternately cycling and walking with $15 \%$ slope (Trac 3000 Tour Med and Crosstrainer 3000; Ergo-Fit Inc., Pirmasens, Germany) (see Fig. 1). To simulate an altitude of $3883 \mathrm{~m}$ a.s.l., participants were exposed to an inspiratory oxygen fraction of $13.1 \%$ at constant room temperature $\left(20-24{ }^{\circ} \mathrm{C}\right)$ and humidity $(20-27 \%)$ for $4 \mathrm{~h}$.

\section{Acute mountain sickness score and cognitive performance}

Symptoms of AMS, consisting of headache, gastrointestinal problems, insomnia, fatigue and dizziness, were evaluated using a self-report questionnaire according to the Lake Louise Score (LLS, 5 items, maximum point sum 15) [12]. AMS after exposition to $\mathrm{NH} / \mathrm{HH}$ was defined as presence of moderate or severe headache in combination with a LLS point sum of $\geq 3$. Cognitive function was evaluated on an Android tablet with a test battery developed by the Mobile Health Systems Lab, Eidgenössische Technische Hochschule (ETH), Zurich, Switzerland, with a total of 4 different cognitive tests: first, Trail Making Test A (TMT-A), where subjects must connect numbers and Trail Making Test B (TMT-B), where subjects must connect numbers and letters in an ascending sequence (i.e. 1-A, 2-B, 3-C...) as quickly as possible. Second, a target reaction test (tRT) and a sorting reaction test (sRT) were performed. In the tRT, one must keep a finger on a predefined area of the tablet until a spot appears which should be touched as quickly and accurately as possible. In the sRT, similar looking geometrical forms must be quickly touched in the order displayed above. For all cognitive tests, speed, accuracy and response time were recorded electronically. Subjects were asked to take 3 of each test type in an isolated environment. Prior to the study, individuals trained all tests to become familiar with the test battery and handling of the tablet.

\section{Cerebral oxygenation and advanced hemodynamic monitoring}

All variables regarding hemodynamics, peripheral oxygen saturation and cerebral oxygenation were repeated five-times at each time point to calculate mean values for every subject. Heart rate, peripheral oxygen saturation and non-invasive blood pressure were measured with a mobile battery powered monitoring system (Infinity ${ }^{\circ} 540$ Monitoring, Draeger Inc., Luebeck, Germany). Cerebral oxygenation $\left(\mathrm{rScO}_{2}\right)$ was measured using a noninvasive near-infrared spectroscopy (NIRS) monitor (INVOS $^{\text {mi }}$ 5100C Cerebral/Somatic Oximeter, Covidien G, Boulder, CO, USA) powered by battery and a portable $240-\mathrm{V}$ power converter. Non-invasive advanced hemodynamic monitoring was performed with a portable monitor using electrical cardiometry $\left(\mathrm{ICON}^{\mathrm{wa}} \mathrm{Car}-\right.$ diac Output Monitor, Osypka Medical GmbH, Berlin, Germany) to measure cardiac index (CI), stroke volume (SV), index of contractility (ICON) and left-ventricular ejection time (LVET). This technique is based on variations of thoracic electrical bioimpendance due to changes in thoracic conductivity during the heart cycle registered by highly conductive sensors (Cardiotronic Sensors $^{\text {Th }}$ Osypka Medical GmbH, Berlin, Germany) [13].

\section{Statistical analysis}

Normally distributed data are given as mean and standard deviation. In case of repeated measurements, a one-way ANOVA with Greenhouse-Geisser correction, followed by multiple comparisons with Bonferroni correction was performed $(p<0.05 / \mathrm{n})$. Differences of LLS were analyzed by Wilcoxon matched-pairs test. Correlations were assessed using Spearman's rank correlation coefficients. T-test with Bonferroni-Sidak correction was used to detect differences between groups after exercise. All statistical analyzes were performed using PRISM version 7 (GraphPad Software Inc., La Jolla, CA, USA).

\section{Results}

Compared to baseline, systemic and cerebral oxygen saturation after exercise decreased significantly under normobaric and hypobaric conditions $\left(\mathrm{NH}: \mathrm{SpO}_{2} 97.7 \pm\right.$ $1.5 \%$ versus $82.9 \pm 5.8 \%, p<0.01 ; \mathrm{rScO}_{2} 73.7 \pm 6.0 \%$ versus $62.0 \pm 5.6 \%, \mathrm{p}<0.05 ; \mathrm{HH}: \mathrm{SpO}_{2} 96.7 \pm 1.0 \%$ versus $84.8 \pm 4.9 \%, p<0.001 ; \mathrm{rScO}_{2} 73.4 \pm 8.3 \%$ versus $56.3 \pm$ $11.0 \%, p<0.001$ ) (see Fig. 2a and b). After $24 \mathrm{~h}$ in hypobaric hypoxia 6 of 11 subjects (54.6\%) developed AMS (moderate or severe headache in combination with LLS point sum $\geq 3$ ). LLS point sum increased significantly after exercise in $\mathrm{NH}$ and $\mathrm{HH}$ and highly significantly after $24 \mathrm{~h}$ in $\mathrm{HH}$ compared to baseline measurements (see Fig. 2c). However, cognitive function tests at either $\mathrm{NH}$ or $\mathrm{HH}$ remained unchanged and did not correlate with changes in hemodynamics, oxygen saturation or 


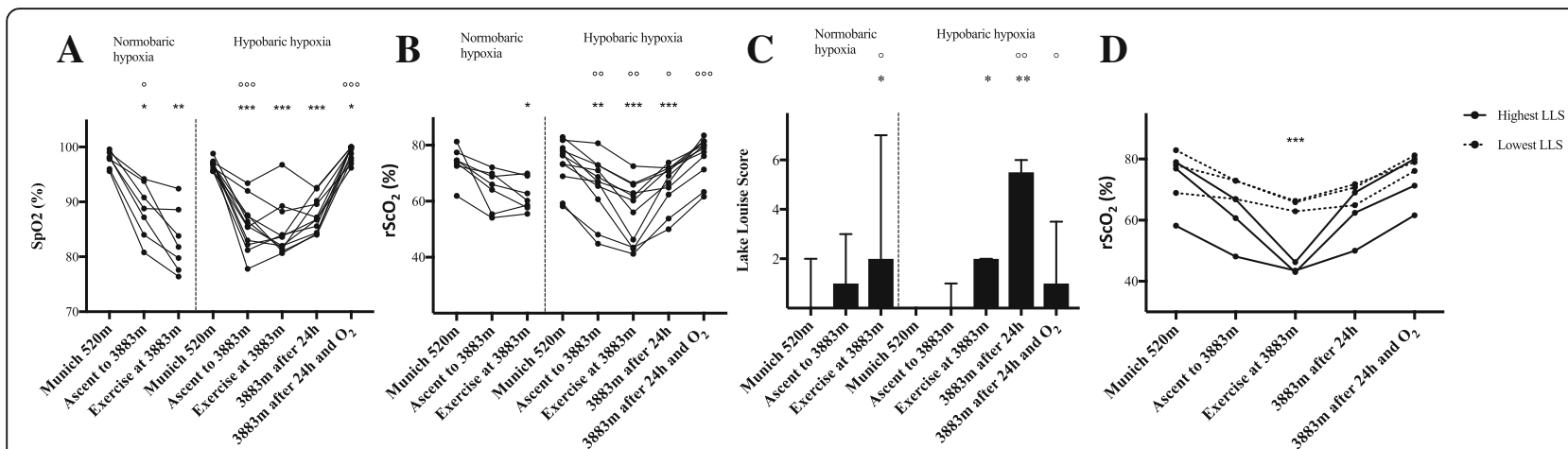

Fig. 2 Peripheral and cerebral oxygen saturation and Lake Louise Score in normobaric (hypoxic chamber; $n=7$ ) and hypobaric (Little Matterhorn summit; $n=11)$ hypoxia. a Changes in pulse oximetry saturation $\left(\mathrm{SpO}_{2}: \%\right)$. b Changes in regional cerebral oxygen saturation $\left(\mathrm{rScO}_{2} \%\right.$ mean values of right and left hemisphere). Statistical analysis for A and B using one-way repeated-measures ANOVA with Greenhouse-Geisser correction, followed by multiple comparisons with Bonferroni correction. c Changes in Lake Louise Score (LLS: median and interquartile-range) for evaluation of Acute Mountain Sickness. Subjects completed a self-reported questionnaire on a tablet. Statistical analysis using Wilcoxon matchedpairs signed rank tests. $\mathbf{d}$ Comparison of regional cerebral oxygen saturation $\left(\mathrm{rSCO}_{2}: \%\right.$; mean values of right and left hemisphere) between the four subjects with lowest LLS and the four subjects with highest LLS after $24 \mathrm{~h}$ in hypobaric hypoxia. Statistical analysis using t-test with Bonferroni-Sidak correction, difference between groups after exercise ${ }^{*} p<0.05,{ }^{* *} p<0.01,{ }^{* * *} p<0.001$ vs. baseline in Munich at $520 \mathrm{~m}^{\circ}{ }^{\circ} p<0.05,{ }^{\circ 0} p<0.01,{ }^{\circ 00} p<$ 0.001 vs. prior time point

LLS. Comparing the four subjects with highest and lowest LLS in $\mathrm{HH}$, significant differences in $\mathrm{rScO}_{2}$ between groups were revealed ( $p<0.01$; see Fig. $2 \mathrm{~d}$ ). During exercise $\mathrm{HR}$ was $133.3 \pm 16.9 \mathrm{~min}^{-1}$ in $\mathrm{NH}$ compared to $142.8 \pm 20.5 \mathrm{~min}^{-1}$ in $\mathrm{HH}(p=0.002)$. After exercise, HR remained increased in both $\mathrm{NH}\left(61.6 \pm 8.5 \mathrm{~min}^{-1}\right.$ versus $\left.87.7 \pm 7.8 \mathrm{~min}^{-1} ; p<0.01\right)$ and HH $\left(61.1 \pm 8.8 \mathrm{~min}^{-1}\right.$ versus $\left.101.8 \pm 6.8 \mathrm{~min}^{-1} ; p<0.001\right)$ compared to baseline measurements, accompanied by a simultaneous decrease of LVET whereas mean arterial pressure only increased in hypobaric conditions $(86.6 \pm 5.4 \mathrm{mmHg}$ versus $98.8 \pm$ $8.9 \mathrm{mmHg} ; p<0.05$ ) compared to baseline (see Figs. 3a, b and c). Cardiac index increased significantly after exercise at high altitude (see Fig. 3d). Further values are given at
Table 1. In $\mathrm{HH}$, a significant negative correlation was found between HR and LLS $(r=-0.692 ; p<0.05)$. Additionally, after exercise, $\mathrm{rScO} 2$ correlated with $\mathrm{HR}$ $(r=0.802 ; p<0.01)$, CI $(r=0.682 ; p<0.05)$ and SV $(0.709 ; p<0.05)$. Furthermore, in $\mathrm{HH}, \mathrm{rScO}_{2}$ after exercise showed an inverse correlation with LLS after $24 \mathrm{~h}(r=-0.817 ; p<0.01)$. Most importantly, reduced $\mathrm{rScO} 2$ after exercise in $\mathrm{NH}$ was inversely correlated with LLS after $24 \mathrm{~h}$ on the mountain $(r=-0.971 ; p<0.01)$.

\section{Discussion}

Acute mountain sickness is an ongoing topic in high altitude medicine. Until now, different variables with a predictive value for AMS could be identified $[6,7]$. Of

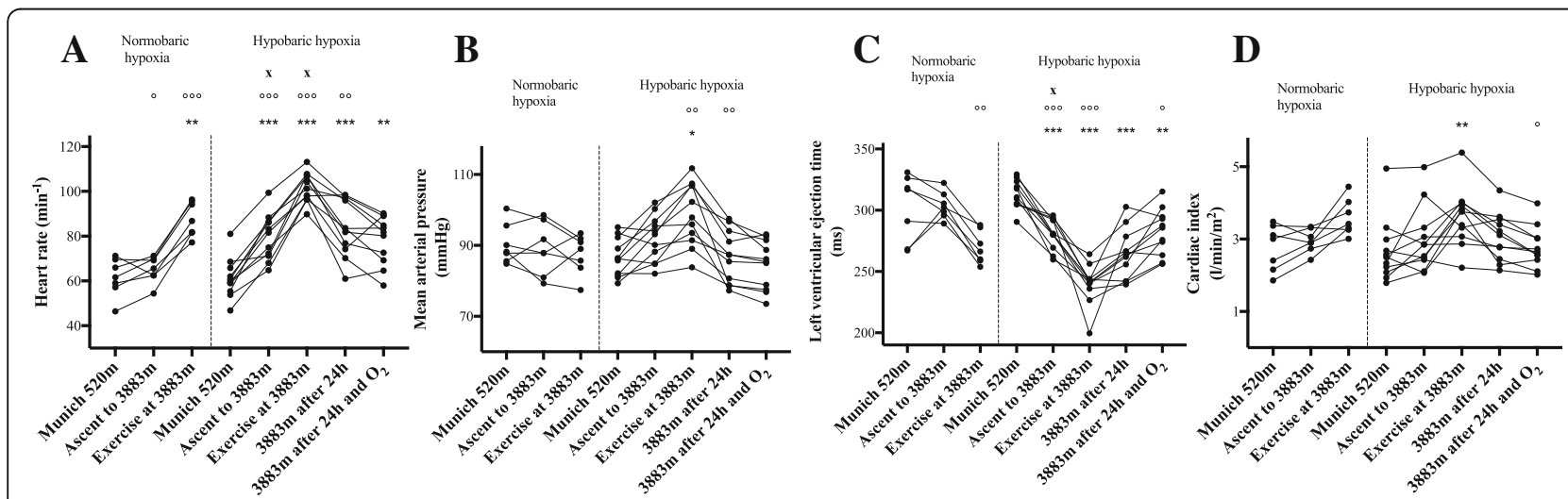

Fig. 3 Hemodynamic variables in normobaric (hypoxic chamber; $n=7$ ) and hypobaric (Little Matterhorn summit; $n=11$ ) hypoxia. Statistical analysis using one-way repeated-measures ANOVA with Greenhouse-Geisser correction, followed by multiple comparisons with Bonferroni correction. a Changes in heart rate $\left(H R ; \mathrm{min}^{-1}\right)$. b Changes in mean arterial pressure (MAP; $\mathrm{mmHg}$ ). $\mathbf{c}$ Changes in left ventricular ejection time (LVET; ms). $\mathbf{d}$ Changes in cardiac index $\left(\mathrm{Cl} ; \mathrm{l} / \mathrm{min} / \mathrm{m}^{2}\right) .{ }^{*} p<0.05,{ }^{* *} p<0.01,{ }^{* * *} p<0.001$ vs. baseline in Munich at $520 \mathrm{~m} ;{ }^{\circ} p<0.05,{ }^{\circ} p<0.01,{ }^{\circ 00} p<0.001$ vs. prior time point; $\mathbf{x} p<0.05$ between corresponding time points in hypobaric and normobaric hypoxia $(n=7)$ 
Table 1 Parameters measured with noninvasive hemodynamic monitoring using Electrical Cardiometry

\begin{tabular}{|c|c|c|c|c|c|c|c|c|}
\hline & \multicolumn{3}{|c|}{ Normobaric hypoxia (hypoxic chamber) } & \multicolumn{5}{|c|}{ Hypobaric hypoxia (Little Matterhorn summit) } \\
\hline & $\begin{array}{l}\text { Munich } \\
520 \mathrm{~m}\end{array}$ & $\begin{array}{l}\text { Ascent to } \\
\text { simulated } 3883 \mathrm{~m}\end{array}$ & $\begin{array}{l}\text { Exercise at } \\
\text { simulated } 3883 \mathrm{~m}\end{array}$ & $\begin{array}{l}\text { Munich } \\
520 \mathrm{~m}\end{array}$ & $\begin{array}{l}\text { Ascent to } \\
3883 \mathrm{~m}\end{array}$ & $\begin{array}{l}\text { Exercise at } \\
3883 \mathrm{~m}\end{array}$ & $\begin{array}{l}3883 \mathrm{~m} \\
\text { after } 24 \mathrm{~h}\end{array}$ & $\begin{array}{l}3883 \mathrm{~m} \text { after } \\
24 \mathrm{~h} \text { and } \mathrm{O}_{2} \\
\end{array}$ \\
\hline Cardiac index $\left(1 / \mathrm{min} / \mathrm{m}^{2}\right)$ & $2.8 \pm 0.6$ & $3.0 \pm 0.3$ & $3.6 \pm 0.50$ & $2.7 \pm 0.9$ & $3.0 \pm 0.9$ & $3.6 \pm 0.8^{* *}$ & $3.1 \pm 0.7$ & $2.8 \pm 0.6$ \\
\hline Cardiac output (I/min) & $5.2 \pm 0.8$ & $5.6 \pm 0.5$ & $6.8 \pm 0.8$ & $5.0 \pm 1.5$ & $5.8 \pm 1.6$ & $6.9 \pm 1.5$ & $5.8 \pm 1.4$ & $5.3 \pm 1.2$ \\
\hline Stroke volume (ml) & $84.3 \pm 7.8$ & $85.3 \pm 7.6$ & $76.5 \pm 8.4$ & $81.4 \pm 14.0$ & $74.1 \pm 14.0$ & $68.0 \pm 10.7^{*}$ & $67.6 \pm 13.5^{*}$ & $69.6 \pm 13.6$ \\
\hline $\begin{array}{l}\text { Left ventricular ejection } \\
\text { time (ms) }\end{array}$ & $303 \pm 27$ & $304 \pm 11$ & $267 \pm 13$ & $313 \pm 12$ & $281 \pm 12^{* * *}$ & $240 \pm 16^{* * *}$ & $264 \pm 20^{* * *}$ & $282 \pm 19^{* *}$ \\
\hline Index of contractility & $68.8 \pm 20.1$ & $68.2 \pm 8.0$ & $53.1 \pm 13.8$ & $64.8 \pm 22.6$ & $50.0 \pm 17.5$ & $45.2 \pm 17.1^{* *}$ & $44.0 \pm 15.2^{* *}$ & $45.9 \pm 13.6^{*}$ \\
\hline
\end{tabular}

Values are presented as mean \pm SD. Statistical analysis with one-way repeated-measures ANOVA with Greenhouse-Geisser correction, followed by multiple comparisons with Bonferroni correction for multiple comparisons, ${ }^{*} p<0.05,{ }^{* *} p<0.01,{ }^{* * *} p<0.001$ vs. baseline in Munich at $520 \mathrm{~m}$

high interest, Sutherland et al. have recently shown a significant correlation between heart rate variability and AMS, evaluated by LLS [8]. However, HRV can be of limited value in subjects with cardiovascular comorbidities i.e. arrhythmias or ß-blocker intake. It is therefore of interest to identify further predictive variables which can be assessed easily even in remote areas. In our study we were able to identify further hemodynamic variables associated with AMS. To the best of our knowledge, this is the first trial using electrical cardiometry combined with cerebral near-infrared spectroscopy in high altitude.

In detail, in our healthy volunteers we have seen significant increases in $\mathrm{HR}, \mathrm{CI}, \mathrm{SV}$ and decreases in LVET, ICON and cerebral oxygenation. Effects on hemodynamic variables were most pronounced after exercise in both, normobaric and hypobaric hypoxia at an altitude of $3883 \mathrm{~m}$ a.s.l.. In accordance to the results of Sutherland et al. [8] we found a significant negative correlation between HR and LLS in hypobaric conditions. However, variables assessed in hypobaric conditions could only allow recommendations to interrupt further ascent or to immediately descend but they cannot provide predictive value. In contrast, associations between variables assessed in a safe normobaric training environment and the risk of developing actual AMS at high altitude could help to predict the individual's risk for AMS. In this regard, we could show a negative correlation between normobaric $\mathrm{rScO} 2$ decrease after exercise and LLS after $24 \mathrm{~h}$ in hypobaric conditions on the mountain. Due to the fact that our subjects were the same under $\mathrm{NH}$ and $\mathrm{HH}$, an association is likely. Thus, the $\mathrm{rScO} 2$ decrease in simulated altitude in a normobaric chamber might serve as a predictive variable in the future. This is interestingly due to the fact, that there is actually an existing debate about the air equivalent model, which points out, that $\mathrm{NH}$ and $\mathrm{HH}$ are two different stimuli for AMS $[14,15]$. In some previously published trials, the severity of AMS was higher in $\mathrm{HH}$ than in $\mathrm{NH}$ whereas the underlying mechanism is unclear $[16,17]$. Additionally, preacclimatization in $\mathrm{HH}$ can reduce severity of AMS whereas preacclimatization in $\mathrm{NH}$ was less effective [18-21]. However, the main factor affecting AMS seems to be acclimatization to hypoxia. The role of hypoxia in AMS was also supported by our presented data. When focusing on the four individuals with highest and lowest LLS, it turns out that they had lowest / highest cerebral oxygenation which supports the hypothesis, that oxygen delivery and clinical symptoms are associated. Simultaneously, the significant correlation between the $\mathrm{rScO} 2$ decrease after exercise and corresponding cardiac index underlines the importance of adequate hemodynamic adaptation to hypoxic conditions. Although this is not a new finding, it is of interest that we have found associations between short exercise in a hypoxic chamber and symptoms of AMS in high altitude. Furthermore, subjects who were able to adequately adapt their cardiac index either by an increase of HR or SV, have shown better cerebral oxygenation and lower LLS point sum. Thus, $\mathrm{rScO} 2$ after exercise under normobaric hypoxia could be a possible predictor for AMS at high altitude. This could be interestingly due to the fact that access to high altitude areas is getting easier even for subjects with cardiovascular diseases exposing those individuals at risk for AMS [22].

In contrast to the observed changes in hemodynamics and oxygenation, the cognitive function tests used in this trial did not reveal any changes. This is in some accordance with the literature where results are inconsistent: Asmaro et al. (2013) investigated cognitive dysfunction in hypoxic conditions at a simulated altitude up to $7.620 \mathrm{~m}$ a.s.l. in healthy volunteers [2]. The authors were able to detect impairments of cognitive performance in this setting of extreme high altitude. Davranche et al. (2016) studied brain oxygenation and cognitive function during 4 days at an altitude of $4.350 \mathrm{~m}$ a.s.l. and detected a reduction in terms of speed and accuracy in the early phase of hypoxic exposure whereas the slowdown of reaction time was not detectable anymore after 2 days at high altitude [3]. However, Issa et al. (2016) found no significant changes in overall cognitive performance during an expedition to Mount Everest [23]. Also, Pramsohler et al. (2017) described inconsistent finding regarding cognitive 
function in subjects that slept at a simulated altitude of $5.500 \mathrm{~m}$ a.s.l. [1]. While the combined parameter of cognitive- and motoric reaction time didn't change, these authors even found a correlation between lower $\mathrm{SpO}_{2}$ and shorter cognitive reaction time. In summary, the data regarding hypoxia and cognitive function are contradictory. One reason for this could be the fact that the tests applied throughout the studies are not standardized and vary. In any case, at this point, cognitive function tests are not associated with symptoms of AMS.

Our study has limitations: First, we only included healthy volunteers and can only speculate that the cerebral oxygenation decrease in normobaric hypoxia would be of predictive value in patients with decreased heart rate variability. Secondly, due to the higher heart rate, exercise intensity seems to be slightly higher in $\mathrm{HH}$ than in $\mathrm{NH}$. This is probably due to the fact that the expedition on the glacier had not been carried out as originally planned due to the weather conditions, but had to be modified. Third, the set of cognitive function tests used was insensitive to detect mild cognitive impairment. Thus, in future studies, a larger set of more standardized tests is recommended. However, our trial provides new insights regarding the relation between hemodynamics, cerebral oxygenation and LLS, and thus these variables assessed in normobaric conditions might help to predict AMS in high altitude.

\section{Conclusion}

Non-invasive hemodynamic variables and cerebral oxygenation after exercise in normobaric hypoxia seem to be associated with the occurrence of acute mountain sickness at high altitude. This could be particularly interesting as a predictor for acute mountain sickness. The variables described here for the first time should therefore be investigated further in high altitude including more healthy participants as well as subjects with comorbidities.

\section{Acknowledgements}

Especially, we want to thank Jutta Stephan from the Institut für Höhentraining, Munich, Germany (http://www.hoehenbalance-muenchen.de) for providing access to the normobaric chamber and expertise.

\section{Ethical approval and consent to participate}

Ethical approval was given by the local Ethics Committee of the University of Munich, Germany (project no. 350-16). Written informed consent was given prior to the study by all participants.

\section{Funding}

The work was supported in part by a research grant to Tobias Kammerer by Deutsche Gesellschaft für Berg- und Expeditionsmedizin e.V.. Monitors and electrodes for cerebral oximetry measurements were sponsored by Covidien Deutschland $\mathrm{GmbH}$, Medtronic company.

\section{Availability of data and materials}

The datasets used and analyzed during the current study are available from the corresponding author on reasonable request.

\section{Authors' contributions}

TK, VF and STS have written the manuscript. TK is the main author. TK, VF, $A B, F B, J M K, M R, C S$ and STS have planned the experimental normobaric and hypobaric trial protocol. TK, VF, NH, AB, FB, JMK, CS and STS were responsible for blood samples, hemodynamic monitoring, cognitive function tests and medical care during the mountain expedition. MH, TK and VF have done statistical analyses and graphs. WK has developed and analyzed cognitive function tests. All authors read and approved the final manuscript.

\section{Consent for publication}

All authors have given their consent for publication in this journal. Written informed consent was obtained from the participants for publication of their individual details and accompanying figures in this manuscript. The consent form is held by the authors and is available for review by the Editor-in-Chief.

\section{Competing interests}

The authors declare that they have no competing interests.

\section{Publisher's Note}

Springer Nature remains neutral with regard to jurisdictional claims in published maps and institutional affiliations.

\section{Author details}

'Department of Anaesthesiology, University Hospital, LMU Munich, Marchioninistr. 15, 81377 Munich, Germany. ${ }^{2}$ Walter Brendel Centre of Experimental Medicine, LMU Munich, Marchioninistr. 15, 81377 Munich, Germany. ${ }^{3}$ Exercise Physiology Lab, Institute of Human Movement Sciences and Sport, ETH Zurich, Winterthurerstr. 190, 8057 Zurich, Switzerland. ${ }^{4}$ Mobile Health Systems Lab, Institute of Robotics and Intelligent Systems, ETH Zurich, Lengghalde 5, 8092 Zurich, Switzerland. ${ }^{5}$ Institute of Anesthesiology, Heart and Diabetes Center NRW, Ruhr University Bochum, Georgstr. 11, 32545 Bad Oeynhausen, Germany.

Received: 10 September 2018 Accepted: 31 October 2018

Published online: 19 November 2018

\section{References}

1. Pramsohler S, Wimmer S, Kopp M, Gatterer H, Faulhaber M, Burtscher M, et al. Normobaric hypoxia overnight impairs cognitive reaction time. BMC Neurosci. 2017;18(1):43.

2. Asmaro D, Mayall J, Ferguson S. Cognition at altitude: impairment in executive and memory processes under hypoxic conditions. Aviat Space Environ Med. 2013;84(11):1159-65.

3. Davranche K, Casini L, Arnal PJ, Rupp T, Perrey S, Verges S. Cognitive functions and cerebral oxygenation changes during acute and prolonged hypoxic exposure. Physiol Behav. 2016;164(Pt A):189-97.

4. de Aquino Lemos V, Antunes HK, dos Santos RV, Lira FS, Tufik S, de Mello MT. High altitude exposure impairs sleep patterns, mood, and cognitive functions. Psychophysiology. 2012;49(9):1298-306.

5. Canoui-Poitrine F, Veerabudun K, Larmignat P, Letournel M, Bastuji-Garin S, Richalet JP. Risk prediction score for severe high altitude illness: a cohort study. PLoS One. 2014;9(7):e100642.

6. Richalet JP, Larmignat P, Poitrine E, Letournel M, Canoui-Poitrine F. Physiological risk factors for severe high-altitude illness: a prospective cohort study. Am J Respir Crit Care Med. 2012;185(2):192-8.

7. Schneider M, Bernasch D, Weymann J, Holle R, Bartsch P. Acute mountain sickness: influence of susceptibility, preexposure, and ascent rate. Med Sci Sports Exerc. 2002;34(12):1886-91.

8. Sutherland A, Freer J, Evans L, Dolci A, Crotti M, Macdonald JH. MEDEX 2015: heart rate variability predicts development of Acute Mountain sickness. High Alt Med Biol. 2017;18(3):199-208.

9. Dykiert D, Hall D, van Gemeren N, Benson R, Der G, Starr JM, et al. The effects of high altitude on choice reaction time mean and intra-individual variability: results of the Edinburgh altitude research expedition of 2008. Neuropsychology. 2010;24(3):391-401

10. Komiyama T, Sudo M, Higaki Y, Kiyonaga A, Tanaka H, Ando S. Does moderate hypoxia alter working memory and executive function during prolonged exercise? Physiol Behav. 2015;139:290-6.

11. Virues-Ortega J, Buela-Casal G, Garrido E, Alcazar B. Neuropsychological functioning associated with high-altitude exposure. Neuropsychol Rev. 2004; 14(4):197-224. 
12. Roach R, Bartsch P, Hackett P, Oelz O. The Lake Louise acute mountain sickness scoring system. Hypoxia and molecular medicine. 1993;272:4.

13. Osypka MJ, Bernstein DP. Electrophysiologic principles and theory of stroke volume determination by thoracic electrical bioimpedance. AACN Clin Issues. 1999;10(3):385-99

14. Millet GP, Faiss R, Point PV. Hypobaric hypoxia induces different physiological responses from normobaric hypoxia. J Appl Physiol. 2012; 112(10):1783-4.

15. Conkin J, Wessel JH 3rd. Critique of the equivalent air altitude model. Aviat Space Environ Med. 2008;79(10):975-82.

16. Loeppky JA, Roach RC, Maes D, Hinghofer-Szalkay H, Roessler A, Gates L, et al. Role of hypobaria in fluid balance response to hypoxia. High Alt Med Biol. 2005;6(1):60-71.

17. Roach RC, Loeppky JA, Icenogle MV. Acute mountain sickness: increased severity during simulated altitude compared with normobaric hypoxia. J Appl Physiol. 1996;81(5):1908-10.

18. Beidleman BA, Muza SR, Fulco CS, Cymerman A, Ditzler D, Stulz D, et al. Intermittent altitude exposures reduce acute mountain sickness at $4300 \mathrm{~m}$. Clin Sci (Lond). 2004;106(3):321-8.

19. Fulco CS, Muza SR, Beidleman BA, Demes R, Staab JE, Jones JE, et al. Effect of repeated normobaric hypoxia exposures during sleep on acute mountain sickness, exercise performance, and sleep during exposure to terrestrial altitude. Am J Physiol Regul Integr Comp Physiol. 2011;300(2):R428-36.

20. Stray-Gundersen J, Chapman RF, Levine BD. "Living high-training low" altitude training improves sea level performance in male and female elite runners. J Appl Physiol. 2001;91(3):1113-20.

21. Schommer K, Wiesegart N, Menold E, Haas U, Lahr K, Buhl H, et al. Training in normobaric hypoxia and its effects on acute mountain sickness after rapid ascent to 4559 m. High Alt Med Biol. 2010;11(1):19-25.

22. Levine BD. Going high with heart disease: the effect of high altitude exposure in older individuals and patients with coronary artery disease. High Alt Med Biol. 2015;16(2):89-96.

23. Issa AN, Herman NM, Wentz RJ, Taylor BJ, Summerfield DC, Johnson BD. Association of Cognitive Performance with time at altitude, sleep quality. and Acute Mountain Sickness Symptoms Wilderness Environ Med. 2016; 27(3):371-8

Ready to submit your research? Choose BMC and benefit from:

- fast, convenient online submission

- thorough peer review by experienced researchers in your field

- rapid publication on acceptance

- support for research data, including large and complex data types

- gold Open Access which fosters wider collaboration and increased citations

- maximum visibility for your research: over $100 \mathrm{M}$ website views per year

At $\mathrm{BMC}$, research is always in progress.

Learn more biomedcentral.com/submissions 